\title{
The Research of the Capture Device for PM2.5
}

\author{
Wan Wang \\ College of Chemistry and Chemical Engineering, Guizhou University, Guiyang, Guizhou, China
}

\begin{abstract}
A rigid ceramic filter is an effective Capture Device to remove PM2.5 from gases. In this paper, an experimental system was set up to investigate the effect of operation pressure and flow rate on pressure drop across a Capture Device for PM2.5.
\end{abstract}

\section{Introduction}

With the intensification of processing industries, PM2.5becomes a serious issue. The rigid ceramic filter, such as filter candles, has emerged as one of the most promising choices for hot gases cleaning because of its high filtration efficiency $[1,2]$.The filtration medium is often made of permeable materials with controlled porosity [3]. As the pressure drop is determined by many parameters and operational conditions, still no reliable predictive correlations can be found by now. A lot of studies have been conduced at the laboratorial scale, which can be broadly categorized based on the pulse cleaning system design [1,4-6], the filter candle properties [7], dust properties [8],patchy cleaning [9-11], and so on. In addition, some pilot studies at industrial scale have been carried out where the influence of the face velocity, temperature and pore size of the filter medium was investigated. Chi et al.(2009) has showed that dust deposit within the medium is the main reason for the increase of filter pressure drop. It was recommended that the system should be operatred at low velocity with reasonable ratio of dust size to pore size of the filter medium [12].

In this paper, anther outside operation condition elements will be studied which focus on the operation pressure and flow rate of the inlet dust-laden gases. A series of experiments under different pressure and flow rate will be carried out. In the experiments independent variables, pressure and flow rate, both changed in a liner relation while other conditions are kept the same .The experimental date analysis and result have presented an obvious liner relation between the pressure difference of the membrane, the operation pressure and the flow rate, from which we can obtain an optimal ratio of operation pressure to rate to achieve a better filtration efficiency.

\section{Experiment}

\section{1. experimental system}

The Capture Device for PM2.5 and its Control System was illustrated by Fig.1. Compressive gas was created by a vacuum bump which can provide different pressure gas. The pressure range of compressive gas from 0.1 to $0.9 \mathrm{kgf} / \mathrm{cm} 2$, the detail parameters are showed in Table 1 . The flow rate can be measured by a flow rate meter. Four control valves can be observed in figure1, valve1, 2 and 3 control the inlet gases, respectively, valve4 controls the outlet gas open and close. A constant raw dust concentration was created by a dust feeder, the dust gas stream mixed with the clean gas. Ambient air was drawn through the filter candle by rate pump. The ceramic filter can enter different gases through the control of the three valves. Valvae 3 controls the clean gas at the initial stage for about 10 minutes, during this time the system need to achieve a steady state, with a constant face velocity, pressure and rate.

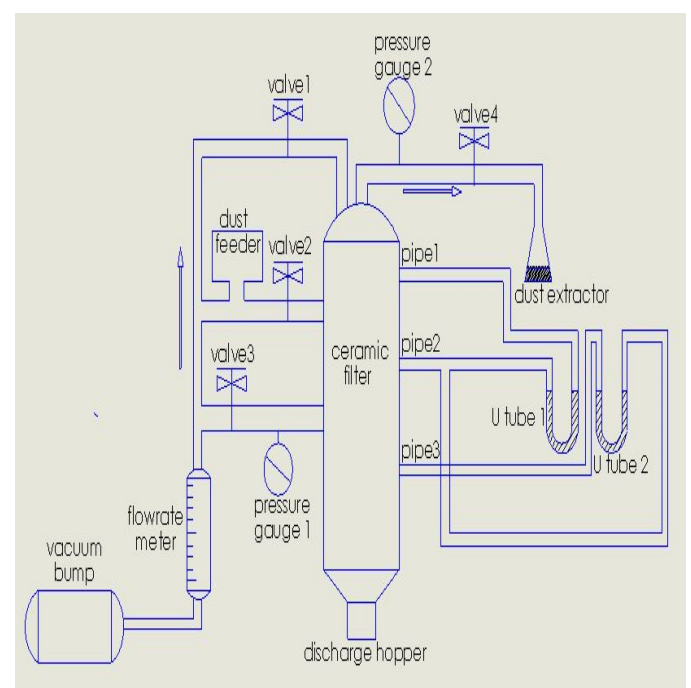

Fig.1 The Capture Device for PM2.5 and its Control System

Then, valve 2 controls dust-laden gas to enter the filter. While the filtration process is going on, valve 3 can control the reverse flow to clean the surface of the filter candle for 30 minutes regularly. The operation pressure in the ceramic filter can be measured by pressure gauge 1. Pipe1 and 3 are connected with the outside of the filter

\footnotetext{
* Corresponding author: wwang7@.gzu.edu.cn
} 
membrane, while pipe 2 is connected with the inside of the membrane. Hence, the reading of the U-tube is the pressure difference between the filter membrane. Account for that the distribution of the pressure within the filter is uneven[13], the average value of U-tube 1 and 2 has been treated as the real pressure difference.

\subsection{Experimental procedure}

A series of experiments were carried out to find the relationship between the pressure difference and the operation pressure, flow rate. The whole process can be divided into two stages, in the first stage, operate pressure is the independent variable, flow rate and other conditions keep constant. While in the second stage, the independent variable is flow rate. Both of the two stages, experiment process should be repeated for many times that data can be achieved as much as possible. Following is the operating process in detail.

At the beginning of the experiment, both valve 2 and 3 were closed. Then valve 3 was opened, it was lasted for 10 minutes. At this stage, the flow pressure and rate were not too high. The gas which entered into the PM 2.5 capture was clean air, which allow the whole system to reach a steady status. After the rate measured by the flow rate meter and the pressure measured by pressure gauge 1 have been stable, then close valve 1 and open valve 3 , dust- laden gases enter the filter. Increase the operation pressure $0.01 \mathrm{MPa}$ every 20 minutes, the pressure can be changed from $0.04 \mathrm{MPa}$ to $0.11 \mathrm{MPa}$. what's more, make sure that the rate, temperature, dust content and all the other conditions to be constant. At the same time, read the two U-tubes every 5 minutes and note down the result. During the process, valve 3 was opened every 20 minutes. The reverse flow was used to clean PM2.5 on the surface of the PM 2.5 Capture.

The second stage has a same operate process as stage 1 , but the independent variable is flow rate.

\section{Result and discussion}

Table 1 and 2 illustrate the pressure difference change with flow rate and operate pressure respectively measured by experiment.

Tab.1The relationship of Pressure difference and flow rate

\begin{tabular}{|c|c|c|}
\hline $\begin{array}{l}\text { Operate } \\
\text { pressure }(\mathrm{MPa})\end{array}$ & Flow rate $\left(\mathrm{m}^{3} / \mathrm{h}\right)$ & $\begin{array}{l}\text { Pressure } \\
\text { difference } \\
\left(\mathbf{D}_{\mathbf{a}}\right)\end{array}$ \\
\hline \multirow{6}{*}{0.08} & 0.8 & 5700 \\
\hline & 0.9 & 6350 \\
\hline & 1 & 6950 \\
\hline & 1.1 & 7500 \\
\hline & 1.2 & 8450 \\
\hline & 0.8 & 6800 \\
\hline
\end{tabular}

\begin{tabular}{|c|c|c|}
\hline 0.1 & 1 & 7400 \\
\cline { 2 - 3 } & 1.036 & 7500 \\
\cline { 2 - 3 } & 1.1 & 9800 \\
\hline
\end{tabular}

Tab.2 Pressure difference change with operate pressure

\begin{tabular}{|c|c|c|}
\hline Flow rate $\left(\mathrm{m}^{3} / \mathrm{h}\right)$ & $\begin{array}{c}\text { Operate } \\
\text { nressure }\end{array}$ & $\begin{array}{c}\text { Pressure } \\
\text { difference }\end{array}$ \\
\hline \multirow{3}{*}{1.036} & 0.04 & 7700 \\
\cline { 2 - 3 } & 0.05 & 7340 \\
\cline { 2 - 3 } & 0.06 & 7050 \\
\cline { 2 - 3 } & 0.08 & 6950 \\
\cline { 2 - 3 } & 0.11 & 6850 \\
\hline \multirow{5}{*}{1.0} & 0.04 & 7500 \\
\cline { 2 - 3 } & 0.06 & 6800 \\
\cline { 2 - 3 } & 0.08 & 6750 \\
\cline { 2 - 3 } & 0.1 & 7400 \\
\hline
\end{tabular}

All these data are measured in temperature about 40 ${ }^{\circ} \mathrm{C}$, ignore the influence of temperature and face velocity. The pressure difference means the differentials between the inside and the outside of the filter membrane.

the pressure difference of the membrane changing with time during the filtration cycle. Four curves are presented in the graph under four different conditions. The initial setting value is $2000 \mathrm{pa}$, it can be found that the pressure differences don't change obviously as time goes on if the operate pressure and rate keep constant, the steep dipping point means reverse flow has affected. The four conditions are the combination of three different pressure and three different rate in order to study the influence of pressure and rate. The stable values are determined by both pressure and flow rate. In addition, a discipline is presented that operate pressure can repress the pressure difference, while the flow rate will contribute to the pressure difference, that is because the highest pressure difference is the result of the case with low operate pressure and high rate.

the influence of the operation pressure to pressure difference, where the flow rate was kept constant. It can be found that with the operate pressure increase the pressure difference displays a drop tendency. Pressure difference presents an inverse proportion to the pressure, this relation can be denoted roughly as equation $\Delta \mathrm{P}=\delta$ $/ \mathrm{P}, \Delta \mathrm{P}$ and $\mathrm{P}$ express the pressure difference within the membrane and operate pressure, respectively. $\delta$ is a parameter can be achieved by experiment, determined by flow rate and other conditions. In order to confirm this relation two different flow rates are used to make a comparison, the change tendency is same, and under the same $\mathrm{P}, \Delta \mathrm{P}$ with higher flow rate is always greater than the one with low flow rate. 


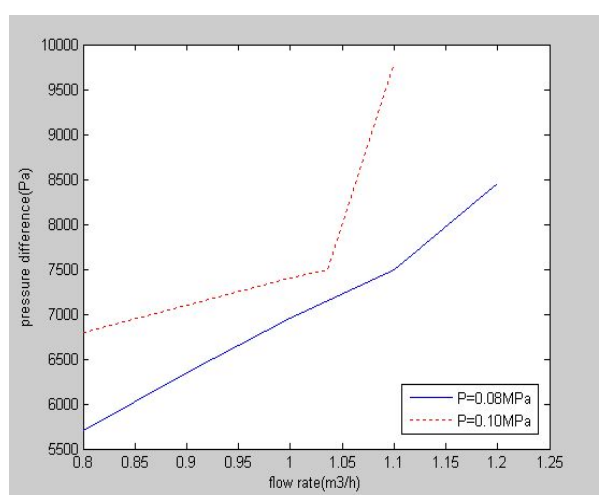

Fig.2 The pressure difference change with flow rate

Fig.2 shows the effect of flow rate to pressure difference, operate pressure keep constant. It can be observed that with the increase of the flow rate, pressure difference also presents a increase tendency, a good liner relation can be found between the two variables. This relation can be denoted as equation $\Delta \mathrm{p}=\left\{\begin{array}{l}a Q+b\left(0<Q \leq Q_{0}\right) \\ c Q+d\left(Q>Q_{0}\right)\end{array}, \quad \Delta \mathrm{P}\right.$ express the pressure difference, $\mathrm{Q}$ express the flow rate, a,b,c,d, $\mathrm{Q}_{0}$ are parameters achieved by experiment and associated with operate pressure and other conditions. Additionally, $\mathrm{Q}_{0}$ is a critical point that when flow rate exceed it then the increase speed of the pressure difference will accelerate. In order to confirm the relation mentioned above, two different operate pressures are used to make a comparison. $\Delta \mathrm{P}$ with higher operate pressure is always smaller than the one with low operate pressure.

\section{Conclusion}

Experiments have shown that both operate pressure and flow rate are responsible for the pressure difference of the filter membrane, they are not affect it lonely but interact with each other. Operate pressure check the increase of the pressure difference, while the flow rate contribute to it.

The relation between the pressure difference, operate pressure and flow rate can be summarized below:

$\mathrm{I}$. Operate pressure is in contrast to pressure difference as equation shows $\Delta \mathrm{P}=\delta / \mathrm{P}$

II. Flow rate is liner with pressure difference, as the increase of flow rate the pressure difference also present an increase tendency as equation shows

$$
\Delta \mathrm{p}=\left\{\begin{array}{l}
a Q+b\left(0<Q \leq Q_{0}\right) \\
c Q+d\left(Q>Q_{0}\right)
\end{array}\right.
$$

Further expand result on this study aimed at finding out the relation between operate pressure and flow rate. Additionally, to discover which of the two has a more important effect on the pressure difference and build a model of the three to make a quantitative analysis. What's more, the parameter list that contains the optimal operate pressure and flow rate was produced. It will help the device to achieve the highest capture efficiency.

\section{Acknowledgement}

The paper was fund by Guizhou science and technology cooperation plan, The Research of the Capture Device for PM2.5 and its Control System, Qiankehe LH zi[2015] 7659.

\section{References}

1. Ji, Z.L., Shi, M.X., Ding, F.X., "Transient flow analysis of pulse-jet generating system in ceramic filter", Powder Technol., 139, 200--207 (2004).

2. Seville, J.P.K., Chuah, T.G., Sibanda, V., Knight, P., "Gas cleaning at high temperatures using ceramic filters", Adv. Powder Technol., 14 (6), 657--672 (2003).

3. B. L. Krasnyi, V. P. Tarasovskii, and A. Yu. Val'dberg, "CERAMIC FILTERS AND THEIR USE FOR CLEANING DUST-LADEN HOT EXIT GASES, Refractories and Industrial Ceramics Vol. 46, No. 2, 2005

4. Choi, J.H., Seo, Y.G., Chung, J.W., "Experimental study on the noz-zle effect of the pulse cleaning for the ceramic filter candle", Powder Technol., 114, 129135 (2001).

5. Chi, H.C., Yu, L., Choi, J.H., Ji, Z.L., “Optimization of nozzle design for pulse cleaning of ceramic filter", Chin. J. Chem. Eng., 16 (2), 306-313 (2008).

6. Ji, Z.L., Peng, S., Tan, L.C., "Numerical analysis of flow field in ceramic filter during pulse cleaning", Chin. J. Chem. Eng., 11 (6), 626-632 (2003).

7. Pastila, P., Helanti, V., Nikkilä, A.P., Mäntylä, T., "Environmental effects on microstructure and strength of SiC-based hot gas filters", J. Eur. Ceram. Soc., 21, 1261-1268 (2001).

8. Alvin, M.A., "Characterization of ash and char formations in advanced high temperature particulate filtration systems", Fuel Process. Technol., 44, 237-283 (1995).

9. Duo, W., Kirkby, N.F., Seville, J.P.K., Clift, R., "Patchy cleaning of rigid gas filters (I) A probabilistic model”, Chem. Eng. Sci., 52 (1), 141--151 (1997).

10. Dittler, A., Ferer, M.V., Mathur, P., Djuranovic, P., Kasper, G., Smith, D.H., "Patchy cleaning of rigid gas filters-transient regeneration phenomena comparison of modeling to experiment", Powder Technol., 124, 55--66 (2002).

11. Ji, Z.L., Jiao, H.Q., Chen, H.H., "Image analysis on detachment process of dust cake on ceramic candle filter", Chin. J. Chem. Eng., 13 (2), 178--186- (2005).

12. CHI Huachang, JI Zhongli, SUN Dongmei . and CUI Lishan, Experimental Investigation of Dust Deposit within Ceramic Filter Medium during FiltrationCleaning Cycles, SEPARATION SCIENCE AND 
ENGINEERING ,Chinese Journal of Chemical Engineering, 17(2) 219--225 (2009)

13. T.G. Chuah, C.J. Withers , J.P.K. Seville, Prediction and measurements of the pressure and velocity distributions in cylindrical and tapered rigid ceramic filters, Separation and Purification Technology 40 (2004) 47-60 